\title{
Leukemia inhibitory factor as an intrapituitary regulator of pro-opiomelanocortin gene expression and corticotrope ontogeny
}

\author{
Laurence Guignat and Jérôme Bertherat \\ Clinique des Maladies Endocriniennes et Métaboliques de l'Hôpital Cochin and Groupe d'Etude en Physiopathologie Endocrinienne, CNRS UPR1524, \\ ICGM, CHU Cochin, Paris, France \\ (Correspondence should be addressed to J Bertherat, CHU Cochin, 27 Rue du FG-ST-Jacques, 75014 Paris, France)
}

\begin{abstract}
Along with the well characterized control of pituitary function by hypothalamic hormones, several cytokines mediate signaling between the neuroendocrine and immune systems. Interleukin-1 (IL-1), IL-6, tumor necrosis factor- $\alpha$ and, recently, leukemia inhibitory factor (LIF) and IL-11 $(1,2)$ are the most thoroughly characterized. LIF belongs to the IL- 6 cytokine family, together with IL-6, IL-11, oncostatin M, cilary neurotropic factor and cardiotropin-1. LIF was originally characterized and so named for its ability to promote the differentiation of a mouse myeloid leukemic cell line (3). LIF is a pleiotropic cytokine and has diverse biological activities, including regulation of hematopoietic cell proliferation and differentiation, stimulation of neuronal growth and development, bone formation, and preparation of the uterus for embryo implantation. Melmed and his colleagues have shown that LIF participates in regulating gene expression of pro-opiomelanocortin (POMC), in addition to corticotropin-releasing hormone (CRH) and glucocorticoids (4-6).
\end{abstract}

LIF gene expression has been demonstrated in human fetal and adult pituitary cells (4), murine corticotrope tumor AtT20 cells (5), and murine hypothalamus and pituitary (7), where it was constitutively expressed and induced in response to lipopolysaccharide endotoxin (7) and IL-1 $\beta$ (2). In AtT20 cells, LIF induces POMC promoter activity, POMC mRNA expression, and adrenocorticotropin (ACTH) secretion. CRH acts synergistically with LIF to stimulate POMC gene expression, whereas glucocorticoids blunt LIF-induced ACTH secretion (4-6). LIF induces ACTH secretion in vitro in primary cultured rat (8) and sheep (9) pituitary cells, and in vivo in fetal monkeys (10) and in mice (7). LIF null mice exhibit an attenuated hypothalamo-pituitary-adrenal (HPA) axis response to stress, especially to chronic inflammatory stress (7) and IL-1 (2), which is restored by delivery of LIF. However, the basal corticosterone plasma level and the response to psychological stress appear intact.

In vitro, LIF induces a switch in proliferating corticotrope tumor AtT20 cells towards a more differentiated phenotype (11). In vivo, transgenic mice expressing LIF driven by the rat growth hormone promoter exhibit persistent Rathke's cysts and somatotrope deficiency
(11). LIF transgenic mice expressing LIF driven by the pituitary glycoprotein hormone $\alpha$-subunit promoter (6) exhibit mild morphological and biochemical features of hypercorticosteronism consistent with Cushing's syndrome associated with corticotrope hyperplasia, deficiency of the other pituitary lineages and multiple Rathke-like cysts. Inhibitory effects on the other axis may, in part, be secondary to LIF-induced hypercorticosteronism. It was suggested that LIF could divert progenitor cells to differentiate from Lhx3-dependent cell lineages (gonadotrope, thyrotrope, somatotrope and lactotrope) to an Lhx3-independent cell lineage (corticotrope), as indicated by the marked reduction in Lhx3 and Pit1 expression, and favor differentiation into ciliated cells. These findings suggest that, together with specific transcription factors, LIF may participate in pituitary ontogeny.

In a wide variety of cells, LIF binding is associated with gp130 (a transmembrane protein subunit shared by the members of the IL- 6 cytokine family) and LIF receptor subunit heterodimerization. Subsequent to LIF receptor dimerization, Janus kinase (JAK) is activated by tyrosine trans/autophosphorylation which leads to tyrosine phosphorylation, homo- or heterodimerization and nuclear translocation of Stat3 and Stat1 (signal transducers and activators of transcription). Although LIF action occurs mainly through the JAK/Stat pathway, other subcellular signaling pathways are activated by IL-6-related cytokines including the Ras-MAPK pathway, phospholipase C, phosphoinositol 3-kinase, phosphotyrosine phosphatase D, pp120 and insulin receptor substrate-1 (12).

To explore molecular mechanisms of LIF action, AtT20 transformants for mutated Stat3 were developed. These mutants act in a dominant negative manner by blocking endogenous Stat3 tyrosine phosphorylation or Stat3 DNA binding, and cause attenuation of LIF-induced transcriptional activation of the POMC promoter, POMC mRNA levels and ACTH secretion. These results indicate a requirement for Stat3 in mediating LIF induction of POMC expression. Furthermore, LIF induces a negative feedback loop in AtT20 cells and in murine pituitary through SOCS-3 (suppressor of cytokine signaling-3), 
which belongs to a family of cytokine-inducible inhibitors of signaling which has recently been described, together with JAK-binding protein and Stat-induced Stat inhibitors (13).

Stat1 homodimer in DNA binding assays does not appear to be regulated by LIF. This finding is somewhat puzzling given the LIF-activation of Stat $1 \alpha$ (and Stat3) reported previously in AtT20 cells (cited in 6). The Ras-MAPK pathway seems not to be implicated in LIF induction of POMC promoter activity, because neither chemical nor molecular blockade of this pathway affects the LIF effect (12). However, LIF induces ERK2 tyrosine phosphorylation (12), and a serine/threonine kinase inhibitor reduces transcriptional LIF-CRH synergy at the POMC promoter (cited in 2).

Elements of the POMC promoter important for the effect of LIF and for LIF-CRH synergy on POMC transcription remain to be elucidated. The possible interactions between Stat 3 and $\mathrm{CRH}$-activated factors such as CREB (cAMP response element-binding protein), AP-1 (activating protein-1) and Nurr (an orphan nuclear receptor) will be important to explore, as well as cross-talking between these pathways. Recently $\mathrm{Ca}^{2+}$ and MAPK-dependent signaling pathways have been demonstrated to be activated after stimulation of $\mathrm{CRH}-1$ and $\mathrm{CRH}-2 \alpha$ receptors, and may be implicated in the LIF-CRH synergy. Furthermore, there are no data on the effect of glucocorticoids on LIF-induced POMC transcription, whereas it has been suggested that cross-talk may exist between the two pathways and that LIF may function acutely to overcome feedback of elevated glucocorticoids in order to maintain activation of the HPA axis in response to stress (5). Interestingly, abnormal JAK/Stat signaling has been observed in some hematopoietic cancers and the LIF gene is expressed in ACTH-producing adenomas and growth hormone-producing adenomas (but not in non-functioning adenomas) (4). Further studies are needed to demonstrate that LIF may take part in pituitary tumorigenesis.

\section{References}

1 Besedovsky H \& Del Ray A. Immune-neuro-endocrine interactions: facts and hypotheses. Endocrine Reviews 199618 206-228.

2 Auernhammer CJ \& Melmed S. Interleukin-11 stimulates proopiomelanocortin gene expression and adrenocorticotropin secretion in corticotroph cells: evidence for a redundant cytokine network in the hypothalamo-pituitary-adrenal axis. Endocrinology $19991401559-1566$.

3 Reichlin S. What's in a name or what does leukemia inhibitory factor have to do with the pituitary gland? Endocrinology 1999 139 2199-2200.

4 Akita S, Webster J, Ren S-G, Takino H, Said J, Zand O et al. Human and murine pituitary expression of leukemia inhibitory factor. Journal of Clinical Investigation 199595 1288-1298.

5 Li Q-L, Yano H, Ren S-G, Li X, Friedman TC \& Melmed S. Leukemia inhibitory factor (LIF) modulates pro-opiomelanocortin (POMC) gene regulation in stably transfected AtT-20 cells overexpressing LIF. Endocrine 19977 325-330.

6 Yano H, Readhead C, Nakashima M, Ren S-G \& Melmed S. Pituitarydirected leukemia inhibitory factor transgene causes Cushing's syndrome: neuro-immune-endocrine modulation of pituitary development. Molecular Endocrinology 199812 1708-1720.

7 Chesnokova V, Auernhammer CJ \& Melmed S. Murine leukemia inhibitory factor gene disruption attenuates the hypothalamopituitary-adrenal axis stress response. Endocrinology 1998139 2209-2216.

8 Kim D-S \& Melmed S. Stimulatory effect of leukemia inhibitory factor on ACTH secretion of dispersed rat pituitary cells. Endocrine Research 199925 11-19.

9 Schartz J, Ray DW \& Perez FM. Leukemia inhibitory factor as an intrapituitary mediator of ACTH secretion. Neuroendocrinology $19996934-43$.

10 Akita S, Conn PM \& Melmed S. Leukemia inhibitory factor (LIF) induces acute adrenocorticotrophic hormone (ACTH) secretion in fetal rhesus macaque primates: a novel dynamic test of pituitary function. Journal of Clinical Endocrinology and Metabolism 199681 4170-4173.

11 Akita S, Readheat C, Stefaneanu L, Fine J, Tampanaru-Sarmesiu A, Kovacs K et al. Pituitary-directed leukemia inhibitory factor transgene forms Rathke's cleft cysts and impairs adult pituitary function. Journal of Clinical Investigation 199799 2462-2469.

12 Bousquet C \& Melmed S. Critical role for Stat3 in murine pituitary adrenocorticotropin hormone leukemia inhibitory factor signaling. Journal of Biological Chemistry 1999274 10723-10730.

13 Auernhammer CJ, Chesnokova V, Bousquet C \& Melmed S. Pituitary corticotroph SOCS-3: novel intracellular regulation of leukemia-inhibitory-factor-mediated proopiomelanocortin gene expression and adrenocorticotropin secretion. Molecular Endocrinology $199812954-961$. 